\title{
Metabolic Syndrome in Patients with Non- alcoholic Fatty Liver Disease: A Community Based Cross-sectional study
}

Mukesh S. Paudel ${ }^{1}$, Awadhesh Tiwari ${ }^{2}$, Amrendra Mandal ${ }^{3}$, Barun Shrestha ${ }^{4}$, Paritosh Kafle ${ }^{3}$, Baikuntha Chaulagai $^{3}$, Sudhamshu KC ${ }^{5}$

1. Gastroenterology, Lumbini City Hospital, Patan, NPL 2. Radiology, Lumbini Medical College and Teaching Hospital, Palpa, NPL 3. Internal Medicine, Interfaith Medical Center, Brooklyn, USA 4. Gastroenterolgy, Chitwan Medical College, Bharatpur, NPL 5. Hepatology, National Academy of Medical Sciences, New Delhi, USA

Corresponding author: Mukesh S. Paudel,drmuku@gmail.com

\section{Abstract \\ Background}

Non-alcoholic fatty liver disease (NAFLD) is the deposition of fat inside liver cells in the absence of secondary causes. It is considered as a hepatic complication of metabolic syndrome. The metabolic syndrome consists of dyslipidemia, hypertension, diabetes, and obesity. This study aims to determine the prevalence of metabolic syndrome in Nepalese patients with NAFLD from mid-Western part of Nepal.

\section{Method}

This was a descriptive cross-sectional study. Three different sites were chosen in and around Butwal submetropolitan city of Rupandehi district, Nepal. A one-day health camp for the screening of fatty liver disease by ultrasonography (USG) was conducted at these sites. Participants with fatty liver were then classified into three grades by USG and the presence of metabolic syndrome was assessed by the National Cholesterol Education Program Adult Treatment Panel III (NCEP-ATPIII) criteria.

\section{Results}

A total of 385 participants with NAFLD were evaluated. Presence of metabolic syndrome by NCEP-ATPIII criteria was found to be in $57.6 \%$ participants; whereas, at least one component of metabolic syndrome was found in $91.4 \%$ of participants with radiologic features of fatty liver. Higher proportion of patients with NAFLD were males. Increased waist circumference followed by low high-density lipoprotein (HDL) level were the most common components of metabolic syndrome in participants with NAFLD.

\section{Conclusions}

Received 02/11/2019

Review began $02 / 13 / 2019$

Review ended 02/13/2019

Published 02/19/2019

๑) Copyright 2019

Paudel et al. This is an open access

article distributed under the terms of the Creative Commons Attribution License CC-BY 3.0., which permits unrestricted use, distribution, and reproduction in any medium, provided the original author and source are credited.
Metabolic syndrome is common in Nepalese community patients with NAFLD.

Categories: Gastroenterology

Keywords: metabolic syndrome, waist circumference, non-alcoholic fatty liver disease, obesity

\section{Introduction}

Non-alcoholic fatty liver disease (NAFLD) has emerged as an important cause of chronic liver disease. It is defined as the accumulation of fat inside the liver cells and the absence of secondary causes of hepatic fat accumulation (alcohol, drugs, viruses, and others) [1]. Evidence of hepatic steatosis is established either by imaging or histology. The metabolic syndrome consists of dyslipidemia, hypertension, diabetes, and obesity [2]. NAFLD is considered as a hepatic complication of metabolic syndrome [3].

There is shown to be an increased prevalence of NAFLD in people with metabolic syndrome and vice versa. NAFLD has a reported prevalence ranging from $20 \%$ to $30 \%$ depending on the studied populations [4]. The prevalence of metabolic syndrome in NAFLD is shown to be between $50 \%$ to $67 \%$ in various studies [5-6].

There is a paucity of data on the association of metabolic syndrome with NAFLD from our part of the world. The aim of the current study was to determine the prevalence of metabolic syndrome in people with NAFLD.

\section{Materials And Methods}

This was a descriptive cross-sectional study. It was conducted from August 1, 2018 to January 13, 2019. Ethical approval was obtained from the Institutional Review Committee of Lumbini Medical College and Teaching Hospital (IRCLMC) prior to the commencement of the study. Informed written consent was taken 
from the participants. The study population consisted of participants of a one-day health camp conducted on separate days in Pratappur village development committee (VDC) of Nawalparasi district, Shankarnagar in Tilottama Municipality and Belbas of Butwal sub-metropolitan city.

The sample size was calculated using the following formula: $\mathrm{n}=\mathrm{Z} 2 \mathrm{P}(1-\mathrm{P}) / \mathrm{d} 2$; where $\mathrm{n}$ is the sample size, $\mathrm{Z}$ is the statistic corresponding to level of confidence, $\mathrm{P}$ is expected prevalence and $\mathrm{d}$ is precision. Assuming $\mathrm{Z}=$ $95 \%$, prevalence of metabolic syndrome $=50 \%$ and $d=0.05$, the sample size was calculated to be 384 . A nonprobability convenient sampling method was used.

All participants who were 18 years of age and above were screened in these health camps for fatty liver by portable ultrasonography (USG) machine (ATNL/51353A, $3.5 \mathrm{MHz}$ convex probe) by a radiologist with more than 10 years of experience in abdominal USG. The exclusion criteria consisted of regular consumption of alcohol >2 units (20 grams) per day, intake of drugs known to cause fatty liver, and history of viral hepatitis, Autoimmune hepatitis, Primary Biliary Cholangitis or Wilsons disease. All eligible participants were then invited to Lumbini City Hospital, Butwal for further evaluation.

A second ultrasound of abdomen was done by the same radiologist and grading of fatty liver disease was done using USG machine from Toshiba Aplio 400 series (Toshiba, Tokyo, Japan) and convex abdominal probe 3.5 MHz. Fatty liver was classified into three grades [7]. Grade I: increase in echogenicity of liver only, Grade II: echogenic liver obscures the echogenic walls of portal vein branches, and Grade III: echogenic liver not only obscures portal vein branches but also the outline of the diaphragm. Participants underwent detailed physical examination and laboratory examination using Biosystems BTS-350 (Biosystem SA, Barcelona, Spain) and colorimetry. A measuring tape was used to measure waist circumference at the midpoint between the costal margin and anterior superior iliac spine. Any potential source of bias was reduced by using the same instruments in each participant. According to the National Cholesterol Education Program Adult Treatment Panel III (NCEP-ATPIII) definition, metabolic syndrome was said to be present if three or more of the following five criteria were met: waist circumference over 40 inches (men) or 35 inches (women), blood pressure over 130/85 mmHg, fasting triglyceride (TG) level over $150 \mathrm{mg} / \mathrm{dl}$, fasting high-density lipoprotein (HDL) cholesterol level less than $40 \mathrm{mg} / \mathrm{dl}$ (men) or $50 \mathrm{mg} / \mathrm{dl}$ (women) and fasting blood sugar over 100 $\mathrm{mg} / \mathrm{dl}[8]$.

Data were entered in Microsoft Excel. Continuous data were expressed as mean and standard deviation. Categorical data were expressed as numbers and percentage. SPSS software version 22 (SPSS Inc., Chicago, IL, USA) was used to analyze the data. We used unpaired t-test for the analysis of continuous variables and chi-square test or Fisher's exact test for categorical variables. Quantitative variables were not grouped during analysis. $P$ value of $<0.05$ was considered statistically significant.

\section{Results}

A total of 573 participants underwent screening USG at three different health camps. Four hundred and two patients were found to have fatty liver by screening USG. The processes of recruitment loss to follow up is shown in Figure 1.

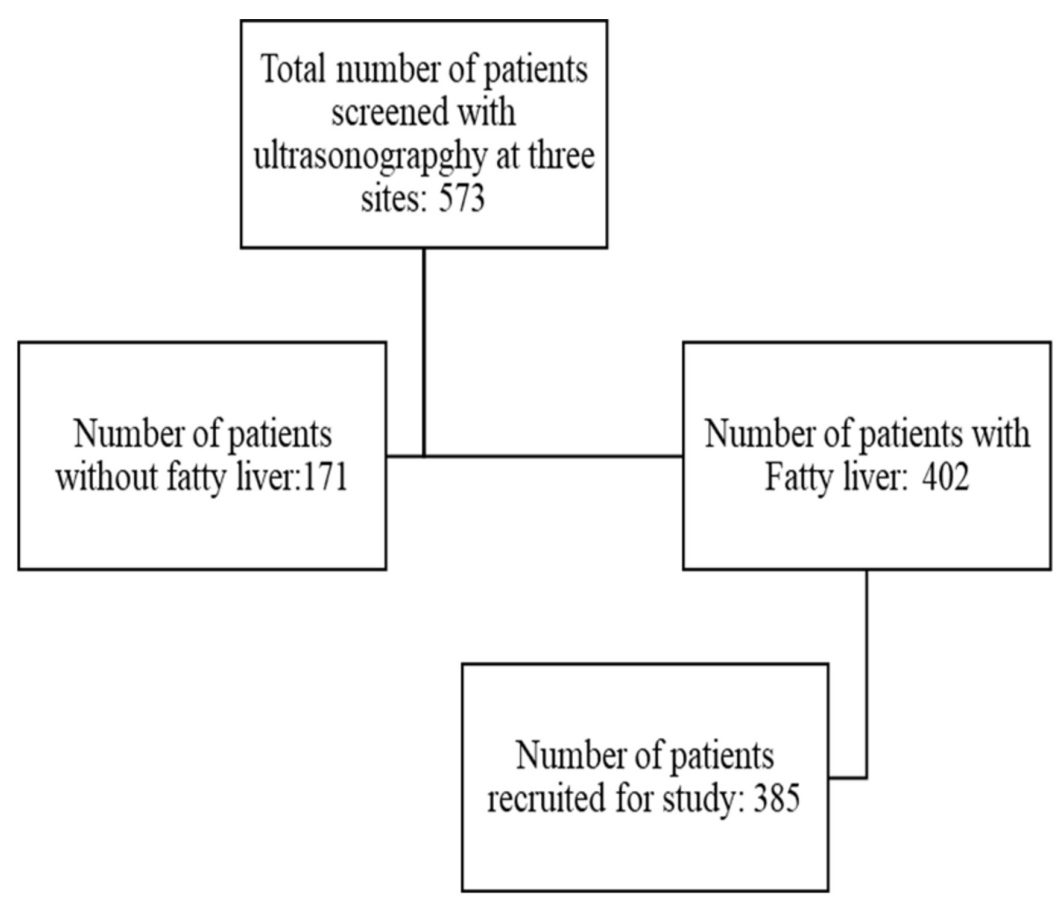


Of the 385 patients that were evaluated at the Lumbini City Hospital and found to have fatty liver by USG, 209 were males and 176 were females. The average age of participants was $44.31 \pm 12.58$ years. Across all grades of fatty liver, the difference in average age of males and females was statistically significant as shown in Table 1.

\begin{tabular}{|c|c|c|c|c|c|c|}
\hline \multirow{2}{*}{ Variable } & \multirow{2}{*}{ Grade } & \multicolumn{2}{|l|}{ Males } & \multicolumn{2}{|l|}{ Female } & \multirow{2}{*}{$p$-value } \\
\hline & & Frequency & Age (years) & Frequency & Age (years) & \\
\hline \multirow{3}{*}{ NAFLD } & I & 131 & $37.17 \pm 7.70$ & 121 & $46.48 \pm 13.17$ & $<0.0001^{\star \star}$ \\
\hline & II & 70 & $43.49 \pm 11.91$ & 53 & $54.45 \pm 10.2$ & $<0.0001^{\star \star}$ \\
\hline & III & 8 & $61.13 \pm 4.55$ & 2 & $74.00 \pm 5.66$ & $0.0085^{\star \star}$ \\
\hline
\end{tabular}

TABLE 1: Frequency and mean \pm standard deviation (SD) of the age of the participants based on different grades of NAFLD

NAFLD: non-alcoholic fatty liver disease

Metabolic syndrome was present in 222 (57.66\%) participants, whereas at least a single component of metabolic syndrome was present in 352 (91.4\%) participants. All five components of metabolic syndrome were present in 41 (10.64\%) participants.

Grade I fatty liver was seen in 65.4\% ( $\mathrm{n}=252, \mathrm{~N}=385)$ participants, Grade II fatty liver in $31.9 \%$ ( $\mathrm{n}=123$, $\mathrm{N}=385)$, and Grade III fatty liver in $2.5 \%(\mathrm{n}=10, \mathrm{~N}=385)$.

Of all the components of metabolic syndrome, most of the patients had increased waist circumference followed by low HDL level as shown in Table 2.

Components of metabolic syndrome

High blood pressure

Raised blood glucose

Increased waist circumference

Raised serum triglycerides level

Low HDL level

\section{Number of patients $n(\%)$}

$197(51.1)$

$176(45.7)$

$258(67.0)$

$232(60.2)$

$239(62.0)$

TABLE 2: Proportion of patients with various components of metabolic syndrome

\section{Discussion}

A total of 385 participants with NAFLD were evaluated in this community-based study and prevalence of metabolic syndrome by NCEP-ATPIII criteria was found to be in $57.6 \%(n=222)$ whereas at least one component of metabolic syndrome was found in $91.4 \%(n=352)$ participants with radiologic features of fatty liver.

In a population-based study on 3,613 patients with NAFLD in the United States, the average age of participants was 43 years which is similar to our study [9]. In a cross-sectional study from Japan, the maximum number of patients with NAFLD were shown to be in the age range of 40 to 49 years [10]. It has also been suggested that males may have a higher prevalence of NAFLD as compared to females and this difference may be related to hormonal factors [11]. Hormonal factors may also be responsible for the difference in age of occurrence of three grades of NAFLD between males and females. Our study also showed a higher proportion of patients with NAFLD to be males ( $n=209,54.28 \%$ ). 
Prevalence of metabolic syndrome in the aforementioned population-based study was shown to be in $67 \%$ of patients [9]; 66\% of males and 65\% of females with NAFLD were found to have metabolic syndrome by NCEP-ATPIII criteria in another study [6]. Studies have shown that $90 \%$ of patients with NAFLD have at least one component of metabolic syndrome and $33 \%$ have all features of metabolic syndrome [12]. All five components of metabolic syndrome were present in 41 (10.64\%) of our study participants. In another hospital-based study from Nepal, the prevalence of metabolic syndrome in patients with NAFLD was found to be $13.6 \%$ with NCEP-ATPIII criteria and $30.1 \%$ with the International Diabetes Federation (IDF) criteria. The difference in the prevalence of metabolic syndrome from our study may be due to the difference in the inclusion criteria [13].

Our study had a slightly higher proportion of patients with Grade I fatty liver as compared to another study from Nepal [14]. The difference may be due to the retrospective nature of their study and the fact that ours was conducted after screening patients in the community.

Metabolic syndrome is an interplay of various factors like obesity, dyslipidemia, dysglycemia, and raised blood pressure [15]. In patients who have NAFLD, the prevalence of obesity ranges from 30\%-100\% [16]. Increased waist circumference was seen in 258 (67\%) of participants in our study. Also, the presence of type 2 diabetes mellitus is seen in $10 \%$ to $75 \%$ of patients with NAFLD [12]. Only 176 (45.7\%) of our study participants with NAFLD had raised blood glucose or diabetes mellitus. Presence of increased serum triglyceride level is seen in 64\% and low HDL levels are seen in 30\% to $42 \%$ of patients with NAFLD [17]. Two hundred and thirty-nine (60\%) patients in our study had raised serum triglyceride and low HDL levels each.

Although NAFLD and metabolic syndrome are closely interrelated, there may be some genetic conditions in which both may not co-exist [15]. We did not evaluate for any genetic polymorphisms for the presence of fatty liver such as Patatin-like phospholipase domain-containing protein 3 (PNPLA3) in our study; and found that 163 (42.4\%) of patients with NAFLD didn't have metabolic syndrome.

Our study had few limitations. We have not looked into the prevalence of underweight patients in those with NAFLD and also didn't use liver biopsy to categorize benign fatty liver vs. non-alcoholic steatohepatitis. Further study may be needed to stratify these higher risk groups in order to treat promptly.

\section{Conclusions}

There is a high prevalence of metabolic syndrome in the Nepalese community with NAFLD. Considering the fact that components of metabolic syndrome are also risk factors of cardiovascular disease, we recommend screening for such factors in patients with seemingly benign finding of fatty liver in USG of the abdomen.

\section{Additional Information \\ Disclosures}

Human subjects: Consent was obtained by all participants in this study. Institutional Review Committee; Lumbini Medical College \& Teaching Hospital Ltd issued approval IRC-LMC 12-E/018. This is to certify that the above mentioned Research Proposal has been reviewed by Institutional Review Committee (IRC-LMC) and approved through review. Animal subjects: All authors have confirmed that this study did not involve animal subjects or tissue. Conflicts of interest: In compliance with the ICMJE uniform disclosure form, all authors declare the following: Payment/services info: All authors have declared that no financial support was received from any organization for the submitted work. Financial relationships: All authors have declared that they have no financial relationships at present or within the previous three years with any organizations that might have an interest in the submitted work. Other relationships: All authors have declared that there are no other relationships or activities that could appear to have influenced the submitted work.

\section{References}

1. Bellentani S: The epidemiology of non-alcoholic fatty liver disease . Liv Int. 2017, 37:81-4. 10.1111/liv.13299

2. Etchegoyen M, Nobile MH, Baez F, et al.: Metabolic syndrome and neuroprotection. Front Neurosci. 2018, 12:96. 10.3389/fnins.2018.00196

3. Bang KB, Cho YK: Comorbidities and metabolic derangement of NAFLD. J Lifestyle Med. 2015, 5:7-13. 10.15280/jlm.2015.5.1.7

4. Al-Dayyat HM, Rayyan YM, Tayyem RF: Non-alcoholic fatty liver disease and associated dietary and lifestyle risk. Diabetes Metab Syndr. 2018, 12:569-75. 10.1016/j.dsx.2018.03.016

5. Moon KW, Leem JM, Bae SS, et al.: The prevalence of metabolic syndrome in patients with nonalcoholic fatty liver. Korean J Hepatol. 2004, 10:197-206.

6. Fattahi MR, Niknam R, Safarpour A, Sepehrimanesh M, Lotfi M: The prevalence of metabolic syndrome in non-alcoholic fatty liver disease; a population-based study. Middle East J Dig Dis. 2016, 8:131. 10.15171/mejdd.2016.18

7. Saadeh S, Younossi ZM, Remer EM, et al.: The utility of radiological imaging in nonalcoholic fatty liver disease. Gastroenterology. 2002, 123:745-50. 10.1053/gast.2002.35354

8. Grundy SM, Cleeman JI, Daniels SR, et al.: Diagnosis and management of the metabolic syndrome: an 


\section{Cureus}

American Heart Association/National Heart, Lung, and Blood Institute scientific statement. Circulation. 2005, 112:2735-52. 10.1161/CIRCULATIONAHA.105.169404

9. Golabi P, Otgonsuren M, de Avila L, Sayiner M, Rafiq N, Younossi Z: Components of metabolic syndrome increase the risk of mortality in nonalcoholic fatty liver disease (NAFLD). Medicine. 2018, 97:e0214. 10.1097/MD.0000000000010214

10. Bertolotti M, Lonardo A, Mussi C, et al.: Nonalcoholic fatty liver disease and aging: epidemiology to management. World J Gastroenterol. 2014, 20:14185-14204. 10.3748/wjg.v20.i39.14185

11. Lonardo A, Carani C, Carulli N, Loria P: 'Endocrine NAFLD' a hormonocentric perspective of nonalcoholic fatty liver disease pathogenesis. J Hepatol. 2006, 44:1196-207. 10.1016/j.jhep.2006.03.005

12. Paschos P, Paletas K: Non alcoholic fatty liver disease and metabolic syndrome. Hippokratia. 2009, 13:9.

13. Pardhe BD, Shakya S, Bhetwal A, et al.: Metabolic syndrome and biochemical changes among non-alcoholic fatty liver disease patients attending a tertiary care hospital of Nepal. BMC Gastroenterol. 2018, 18:109. 10.1186/s12876-018-0843-6

14. Khadka B, Shakya RM, Bista Y: Non-alcoholic fatty liver disease assessment in Nepal . Int J Community Med Public Health. 2016, 3:1654-59. 10.18203/2394-6040.ijcmph20161645

15. Kim D, Touros A, Kim WR: Nonalcoholic fatty liver disease and metabolic syndrome. Clin Liver Dis. 2018, 22:133-40. 10.1016/j.cld.2017.08.010

16. Angulo P: Nonalcoholic fatty liver disease. N Engl J Med. 2002, 346:1221-31. 10.1056/NEJMra011775

17. Marchesini G, Bugianesi E, Forlani G, et al.: Nonalcoholic fatty liver, steatohepatitis, and the metabolic syndrome. Hepatology. 2003, 37:917-23. 10.1053/jhep.2003.50161 